\title{
The Malone Antegrade Continence Enema (MACE) Principle In Children: Is It Important If the Conduit Is Implanted In the Left or the Right Colon?
}

\author{
Karine F. Meyer, Mauricio Macedo, Humberto S. Filho, Thais R. Pinto, Leonan T. Galvao, \\ Quirino C. Meneses
}

Section of Pediatric Surgery (KFM, TRP, LTG, QCM), Hospital do Servidor Publico Estadual, Section of Pediatric Surgery (MM), Hospital Estadual Infantil Darcy Vargas, Section of Pediatric Surgery (HSF), Santa Casa, Sao Paulo, SP, Brazil

\begin{abstract}
Objective: The aim of the study was to determine which was the optimal side for the conduit to be placed (right or left colon) for antegrade continence enema implantation.

Materials and Methods: Between July 1999 and March 2006, 31 patients underwent the construction of a catheterizable conduit using the Malone principle (MACE) In 22 cases the conduit was re-implanted in the right colon and in 9 cases in the left colon. There were 20 male patients and 11 female patients, with a mean age of 10.23 years. The follow-up period varied from 3 from 83 months (average 25 months). Right and left implantation of the conduit in the colon were compared with regards to the presence of complications, volume of the solution utilized, frequency of colonic lavage, time needed for performing the enema, and degree of satisfaction.

Results: One patient with the conduit in the right colon, using the appendix, lost the mechanism after two month follow-up. Thirty patients remain clean and are all capable of performing self-catheterization. No statistically significant differences were found between the groups regarding the variables studied: complications $(p=1.000)$, solution volume $(p=0.996)$, time required $(p=0.790)$ and patient's rating $(p=0.670)$. The lavage frequency required for patients with the conduit in the right colon may be lower.

Conclusion: The MACE principle was considered effective for treating fecal retention and leaks, independent of the implantation site. The success of this surgery appears to be directly related to the patient's motivation and not to the technique utilized.
\end{abstract}

Key words: fecal incontinence; enema; surgery

Int Braz J Urol. 2008; 34: 206-13

\section{INTRODUCTION}

Different diseases can cause intestinal dysfunction and continuous feces soiling. And for the treatment to be adequately performed, it is of utmost importance that a cause be found and eventually treated. Independent of its origin, intestinal incontinence, especially when it has become chronic, could carry significant emotional and social problems. Therefore, the management of children with continuous fecal soiling is essential to improve quality of life (1). 
Retrograde enema was the treatment of choice until recently when there was a major step forward made by Malone et al. in 1990 (2) with the description of the continent cecal access for antegrade enema. The technical procedure utilized the Mitrofanoff (3) principle for the creation of a continent conduit with the appendix, which is easily catheterizable from a stoma.

In cases where the appendix has previously been removed or is required for urological use, the need to construct a new conduit occurs. This has been achieved by utilizing a cecal flap (4), a sigmoid flap (5) or a segment of the small intestine or colon that has been reconfigured (6) by means of the Yang-Monti principle $(7,8)$.

After the reimplantation of these conduits in the left colon began, doubts emerged regarding the optimal place for reimplanting them: the right colon or the left colon. Calado et al. (5) believed that the left colon was the more physiologically appropriate location, with a decrease in the risk of water absorption as well as a decrease in the time required for enema administration and washout, thereby increasing patient satisfaction and compliance. The enema performed in right colon often is long and tedious for handicapped patients, as the volume of washout from the cecum to the rectum is large, especially in neuropathic bowels prone to dolichosigmoid (5).

The aim of the study was to determine which was the optimal side for the conduit to be placed (right or left colon) for antegrade continence enema implantation.

\section{MATERIALS AND METHODS}

Between July 1999 and March 2006, 31 patients with intestinal dysfunction and continuous feces soiling underwent the construction of a catheterizable conduit using the MACE principle (2).

The criteria for indicating surgical treatment were fecal incontinence not responsive to more conservative measures (dietary modifications and medical treatment); patients who remained clean by performing enemas via the rectal route; the patients and their parents should be motivated to perform the antegrade enema.
There were 20 male patients and 11 female patients, with a mean age of 10.2 years (range: 4 to 17 years). Fourteen children had imperforate anus, twelve had myelomeningocele, three had medullar trauma, one had spina bifida, and one had complex perineal trauma (Table-1).

For the 22 patients with the mechanism reimplanted in the right colon (Table-2), the conduit utilized was the cecal appendix, which was kept in its original position and subjected to an anti-reflux procedure consisting of suturing the walls of the cecum around the appendix. In 16 patients, this conduit was brought to the exterior at the umbilicus and in six, at the right iliac fossa. In five of these children, the appendix was divided into two parts (Figure-1), with concomitant division of its irrigation, in order that its proximal part was utilized for intestinal catheterization and its distal part as a Mitrofanoff conduit (3) for performing intermittent urinary catheterization.

In the nine patients with reimplantation of the mechanism in the left colon (Table-2), the conduit was constructed by utilizing a segment of the sigmoid with its base against the mesenteric margin (5). This was tubularized around a urethral probe and was subjected

Table 1 - Patients with fecal incontinence.

\begin{tabular}{lr}
\hline Diseases & N \\
\hline Imperforate anus & 14 \\
Myelomeningocele & 12 \\
Medullar trauma & 3 \\
Spina bifida & 1 \\
Complex perineal trauma & 1 \\
Total & 31 \\
\hline
\end{tabular}

Table 2 - Patients with serious fecal incontinence according to the surgical technique utilized.

\begin{tabular}{lc}
\hline Technique Utilized & N \\
\hline Appendix with anti-reflux mechanism & 22 \\
Tubularized sigmoid & 9 \\
Total & 31 \\
\hline
\end{tabular}



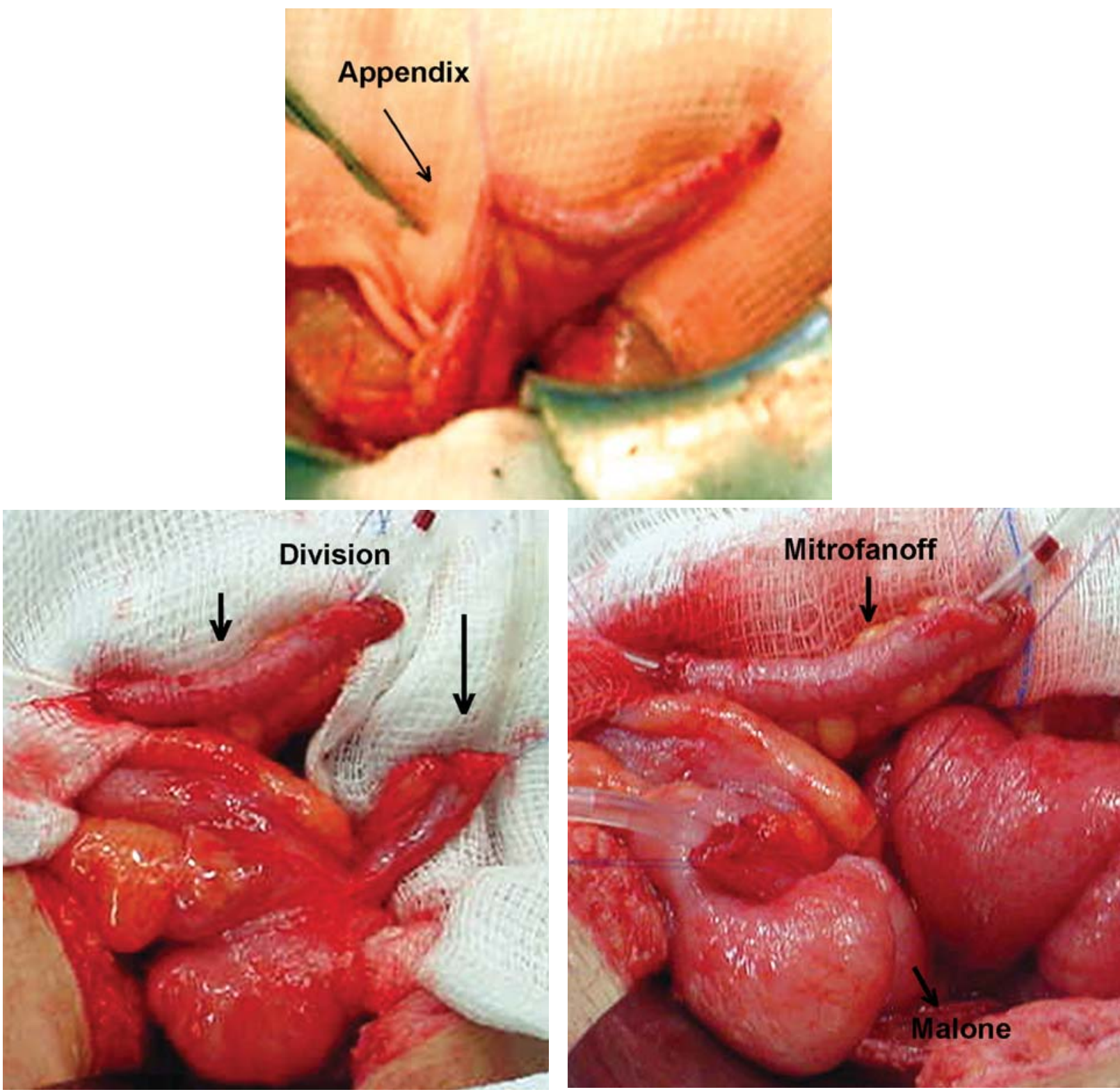

Figure 1 - The appendix was divided into two parts in order that its proximal part was utilized for intestinal catheterization and its distal part as a Mitrofanoff conduit for performing intermittent urinary catheterization.

to an anti-reflux procedure involving the walls of the sigmoid. It was brought to the exterior at the left iliac fossa.

During the same operation, 16 patients simultaneously also underwent other procedures:

- 10 patients: bladder enlargement, construction of a conduit for urinary continence catheterization (Mitrofanoff (3)) and plastic surgery on the bladder neck;
- 2 patients: construction of a conduit for urinary continence catheterization (Mitrofanoff (3));

- 1 patient: sigmoidoplasty to reduce its caliber;

- 1 patient: plastic surgery on the bladder neck;

- 1 patient: reconstruction of the intestinal transit;

- 1 patient: correction of an incisional hernia.

Following the surgery, all the patients were given training, in order to learn how to operate the mechanism. The volume utilized for the enema was 
adjusted individually, so that it would be sufficient for cleaning the colon, without rectal leaks during the day. The solution used depended on the patient's preference (tap water for 14 patients, glycerinated solution for 13 patients and saline solution for 4 patients).

The mean follow-up duration was 25 months (range: 3 to 83 months).

Right and left implantations of the conduit in the colon were compared with regard to the presence of complications, volume of the solution utilized, frequency of enema, time needed for performing the enema, and degree of satisfaction (the patients were asked to give a score on a scale from 1 to 10 to rate the improvement in their quality of life following the construction of the mechanism for performing antegrade enema).

The quantitative variables were represented by mean, standard deviation, median, minimum and maximum, and the qualitative variables by absolute frequency (n) and relative frequency (\%). The non-parametric Mann-Whitney test for independent samples was applied to make comparisons between groups of patients with regard to the variables of solution volume, time needed and patient's rating. Associations between the presence of complications and the side of implantation were evaluated by means of Fisher's exact test. The significance level was set at $0.05(\alpha=5 \%)$.

\section{RESULTS}

One patient had loss of the mechanism after two months follow-up, following presentation of an abscess in the pathway of the appendix that evolved with fibrosis of the conduit. Thirty patients are currently using their conduits: 21 implanted in the right colon and nine in the left colon. All these patients are clean and capable of performing self-catheterization (Table-3).

\section{Complications}

No statistically significant differences were found between the groups $(\mathrm{p}=1.000)$. Six patients $(27.3 \%)$ with the conduit re-implanted in the right colon and constructed using the appendix presented complications:
- stenosis of the stoma in the skin (four patients);

- loss of the mechanism after two months follow-up, following presentation of an abscess in the pathway of the appendix that evolved with fibrosis of the conduit (one patient);

- discharge of secretions (one patient).

Three patients $(33.3 \%)$ with the conduit reimplanted in the left colon and constructed using a sigmoid flap presented complications:

- peristomal infection (two patients);

- difficulty in probing because of angling of the conduit, which necessitated surgical reintervention (one patient);

\section{Volume of the Solution Utilized}

No statistically significant differences were found between the $(p=0.996)$. The patients with the conduit re-implanted on the right side used a mean enema volume of $13.34 \mathrm{~mL} / \mathrm{kg}$. The patients with the conduit re-implanted on the left side used a mean enema volume of $13.35 \mathrm{~mL} / \mathrm{kg}$.

\section{Frequency of Enema}

The patients with the conduit on the left side required daily enema. On the other hand, the enema frequency required for patients with the conduit on the right side may be lower, given that six of our patients $(27 \%)$ performed intestinal cleaning only on alternate days.

\section{Time Needed for Performing the Enema}

No statistically significant differences were found between the groups $(p=0.790)$. The mean time needed for performing the enema was 51.4 minutes (range: 30 to 60 minutes) for the patients with the conduit constructed using the appendix and re-implanted on the right side, and 49.2 minutes (range: 15 to 90 minutes) for the patients with the conduit constructed using the sigmoid and re-implanted on the left side.

\section{Degree of Satisfaction}

No statistically significant differences were found between the groups $(p=0.670)$. The patients were asked to give a score on a scale from 1 to 10 to rate the improvement in their quality of life following the construction of the mechanism for performing antegrade enema. The patients with the conduit in the 
Table 3 - Left colon versus right colon.

\begin{tabular}{|c|c|c|c|c|}
\hline \multirow{2}{*}{$\begin{array}{l}\text { Variables Analyzed } \\
\text { Complications }\end{array}$} & \multicolumn{2}{|r|}{$\begin{array}{l}\text { Right Colon } \\
\qquad(\mathrm{N}=22)\end{array}$} & \multirow[t]{2}{*}{$\begin{array}{l}\text { Left Colon } \\
\qquad(\mathrm{N}=9)\end{array}$} & \multirow{2}{*}{$\frac{\text { p Value }}{1.000}$} \\
\hline & & & & \\
\hline Absent & & $16(72.7 \%)$ & $6(66.7 \%)$ & \\
\hline Present & & $6(27.3 \%)$ & $3(33.3 \%)$ & \\
\hline Solution volume $(\mathrm{mL} / \mathrm{kg})$ & & & & 0.996 \\
\hline mean $( \pm \mathrm{SD})$ & & $13.34(4.91)$ & $13.35(5.62)$ & \\
\hline median & & 12.77 & 13.16 & \\
\hline minimum - maximum & & $5.0-23.8$ & $4.5-21.7$ & \\
\hline Time needed (min) & & & & 0.790 \\
\hline mean $( \pm \mathrm{SD})$ & & $51.4(12.3)$ & $49.2(26.5)$ & \\
\hline median & & 60 & 50 & \\
\hline minimum - maximum & & $30-60$ & $15-90$ & \\
\hline Patient's rating & & & & 0.670 \\
\hline mean $( \pm \mathrm{SD})$ & & $8.9(1.6)$ & $9.2(0.7)$ & \\
\hline median & & 10 & 9 & \\
\hline minimum - maximum & & $5-10$ & $8-10$ & \\
\hline \multirow{3}{*}{ Frequency of lavage } & $\mathrm{N}=15$ & $1 \mathrm{x} /$ day & & \\
\hline & $N=6$ & Alternate days & $1 \mathrm{x} /$ day & NR \\
\hline & $\mathrm{N}=1$ & $2 \mathrm{x} /$ day & & \\
\hline
\end{tabular}

$S D=$ standard deviation.

right colon gave a mean score of 8.9 and the patients with the conduit in the left colon gave a mean score of 9.2 .

\section{COMMENTS}

The MACE procedure has been used in patients with fecal incontinence caused by chronic retention of feces, independent of its etiology, when these patients are resistant to the medical treatment $(5,6,8-10)$. In our study, imperforate anus and myelo- meningocele were the principal diseases presented by the patients.

The procedure, described by Malone et al. (2) in 1990, consists of dissection and isolation of the cecal appendix, suture of the appendix tip into the skin as an appendicostomy, and the implantation of the bottom of the appendix into the cecum in an antireflux tunnel. The appendicostomy is therefore fixed in the inferior right quadrant or in the umbilical region, therefore permitting the introduction of a catheter to perform enemas in an antegrade direction. The original procedure was posteriorly modified, 
maintaining the appendix in its original position and fixing the cecum in the abdominal wall, thus avoiding twisting of the conduit and reducing the time needed to perform the surgery (11).

Mouriquand et al. (6) proposed the use of an isolated intestinal segment of ileum reconfigured using the Yang-Monti $(7,8)$ technique with the implantation of the conduit in the left colon. They reported that this placement of the conduit in the left colon combined with regular performance of enema resulted in the accumulation of feces only in the left colon, reduced time required to perform the enema, and increased child compliance.

Complications involving this procedure are not rare, and the most important ones involve the stoma (12). In the present study, when the conduit utilized was the appendix, stenosis of the skin was the principal complication, and this was corrected by means of dilatation or new maturation of the stoma in the skin. When the conduit utilized was a sigmoid flap, the peristomal infection rate increased, which was expected because of the manipulation involved in opening and suturing colonic loops.

The volumes utilized for performing enemas through conduits implanted on the right and left sides were similar, which has led us to the hypothesis that the volume needed for cleaning the colon is independent from the size of the colon, which is distally situated from the implantation site of the conduit.

If the feces only accumulate in the left colon as stated by Mouriquand et al. (6), one can imagine that even if the conduit is re-implanted in the right colon, it would clear no significant amount of additional feces from the right colon. Thus, the enema volume required for a right-sided conduit could be similar to the volume required for a left-sided conduit.

The enema solution chosen most often by our patients was plain tap water, followed by glycerinated solution and physiological solution. With these solutions, no metabolic disturbances were observed, whereas many have been reported when the solution utilized was phosphated.

There were no differences regarding the time required for performing the enema. The patients took a mean time of 50 minutes (range: 15 to 90 minutes), independent of the conduit reimplantation site.
The patients with the conduit on the left side required daily colon cleaning. On the other hand, the lavage frequency required for patients with the conduit on the right side may be lower, given that six of our patients $(27 \%)$ performed intestinal cleaning only on alternate days.

The authors consider that if the appendix is already available, the MACE procedure can be performed with little mobilization of the organ and the results could be as good as, if not better, than if the conduit was re-implanted directly in the left colon. This has the additional advantage that there is no need to use intestinal segments to perform the catheterization conduit. The appendix should be utilized even when urinary continence derivation is simultaneously necessary, since the proximal appendix can be utilized for intestinal catheterization and the distal appendix can be utilized as a Mitrofanoff conduit (3) for performing intermittent urinary catheterization, as was done in five of our cases, with good results.

The success of this surgery appears to be directly related to the patient's motivation and not to the technique utilized. Thirty patients in this study are currently using their conduits: 21 implanted in the right colon and nine in the left colon. All these patients are clean and are capable of performing self-catheterization. To obtain this high rate of adherence to treatment, all the patients must have been aware that, after the mechanism for antegrade enemas had been constructed, a certain time was needed for adjusting the volume of liquid to be utilized and the frequency of the enemas, until the continence they required was obtained. Through such awareness, abandonment of the use of the conduit was avoided.

When the patients were asked to give a rating from 1 to 10 regarding the improvement in their quality of life following the surgical procedure, the result was a score of 8.9 for the conduits implanted on the right side and 9.2 for the conduits implanted on the left side, thus showing a high satisfaction rate, independent of the implantation site in the colon.

Fecal continence promotes independence among children, and it improves their self-esteem and quality of life. The method utilizing the MACE principle is relatively safe and if we have correctly 
interpreted the results of this limited series, the surgical technique should be the simplest one to perform. Moreover, our patients demonstrated a high degree of satisfaction with the procedure independent of which side it was implanted in.

The success of the Malone procedure seems to depend mainly on selection of the patients. The patients and their parents should be motivated to perform the antegrade enema as a routine task and should be conscious that there is an adaptation period before the expected continence is obtained. Fecal continence promotes the children's independence, improves selfesteem, and improves the quality of life.

The construction of colonic conduit for antegrade enema is not the cure for fecal incontinence but could be an optimal therapeutic option for the patients with chronic and irreversible constipation with soiling. The surgical technique should be the simplest one to perform and our patients demonstrated a high degree of satisfaction with it, independent of which side it was implanted in.

\section{CONFLICT OF INTEREST}

None declared.

\section{REFERENCES}

1. Landman GB, Rappaport L, Fenton T, Levine MD: Locus of control and self-esteem in children with encopresis. J Dev Behav Pediatr. 1986; 7: 111-3.
2. Malone PS, Ransley PG, Kiely EM: Preliminary report: the antegrade continence enema. Lancet. 1990; 336: 1217-8.

3. Mitrofanoff P: Cystostomie continente transappendiculaire. dans le traitement des vessies neurologiques. Chir Pediatr. 1980; 21: 297-305.

4. Kiely EM, Ade-Ajayi N, Wheeler RA. Cecal flap conduit for antegrade continence enema. Br J Surg. 1994: 81: 1215.

5. Calado AA, Macedo A Jr, Barroso U Jr, Netto JM, Liguori R, Hachul M, et al.: The Macedo-Malone antegrade continence enema procedure: early experience. J Urol. 2005; 173: 1340-4.

6. Mouriquand P, Mure PY, Feyaerts A, Morel-Journel N, Jules JA, Basset Debrousse T: The left Monti-Malone. BJU Int. 2000; 85 (suppl 4): 65.

7. Yang WH: Yang needle tunneling technique in creating antireflux and continent mechanisms. J Urol. 1993; 150: 830-4.

8. Monti PR, Lara RC, Dutra MA, de Carvalho JR: New techniques for construction of efferent conduits based on the Mitrofanoff principle. Urology. 1997; 49: 112-5.

9. Dick AC, McCallion WA, Brown S, Boston VE: Antegrade colonic enemas. Br J Surg. 1996; 83: 642-3.

10. Hill J, Stott S, MacLennan I: Antegrade enemas for the treatment of severe idiopathic constipation. $\mathrm{Br} \mathrm{J}$ Surg. 1994; 81: 1490-1.

11. Koyle MA, Kaji DM, Duque M, Wild J, Galansky SH: The Malone antegrade continence enema for neurogenic and structural fecal incontinence and constipation. J Urol. 1995; 154: 759-61.

12. Castellan MA, Gosalbez R, Labbie A, Ibrahim E, Disandro M: Outcomes of continent catheterizable stomas for urinary and fecal incontinence: comparison among different tissue options. BJU Int. 2005; 95: 1053-7.

\section{Correspondence address:}

Dr. Karine Furtado Meyer

Rua Rio Grande, 551/162

São Paulo, SP, 04018-001, Brazil

Fax: + 5511 5084-5342

E-mail: karine_meyer@uol.com.br 


\section{EDITORIAL COMMENT}

The authors have performed a review of their surgical experience with a left- and right-sided Malone antegrade continence enema (MACE) procedure. Their data shows no significant difference in evacuation time, continence, or satisfaction between the two groups.

Interestingly, a significant number of patients with a right MACE were able to irrigate only on alternate days and remain continent as opposed to those with a left MACE. In our institution, a right-sided MACE is nearly always our first choice when using the appendix in its orthotopic location. A Monti-MACE can be implanted wherever it is deemed appropriate from a vascular pedicle standpoint given the restraints of the urinary reconstruction to be performed. A theoretical concern with a left MACE in a myelomeningocele patient with a dilated colon would be proximal constipation that may not be adequately treated by the enema regimen.

Dr. William Robert DeFoor Division of Pediatric Urology Cincinnati Children's Hospital Cincinnati, OH 45229

E-mail: bob.defoor@cchmc.org 